\title{
THE MOVEMENT OF SMALL PARTICULATE MATTER \\ IN THE EARLY SOLAR SYSTEM AND \\ THE FORMATION OF SATELLITES
}

\author{
T. GOLD \\ Center for Radiophysics and Space Research, Cornell University, Ithaca, N.Y., U.S.A.
}

(Read by E. E. Salpeter)

Satellites are a common feature in the solar system, and all planets on which satellite orbits would be stable possess them. (For Mercury the solar perturbation is too large, and the retrograde spin of Venus would cause satellites to spiral in to the planet through tidal friction.) An explanation of the formation of satellites must hence be one which makes the phenomenon exceedingly probable at some stage in the solar system formation processes, and very improbable processes like a capture cannot be the answer in most cases.

Small particulate matter must have been very abundant in the early solar nebula. Such particulate matter must have existed both from the first condensation of the low vapor pressure components of the gas in the first round, and it must also have been composed of material scattered from impacts after some major bodies had begun to form, frequently finding themselves no doubt on collision orbits.

In general, small particulate matter will not follow the same orbits as large bodies would, due to the action of drag forces. In the early solar system such drag must have been present within the original gaseous disc, and at later stages there continued to be a slight drag due to the Poynting-Robertson effect. The gas drag, depending on the mass and temperature distribution of the gas in the early solar nebula, could have acted to supply a force on small particles, either in the forward direction of planetary orbits, or in the retrograde direction. Particles could thus be caused to spiral either outwards or inwards, as a result of such forces.

Such material will frequently not continue to move on a spiral orbit, for it will frequently happen that, as the mean period gradually changes, a particle comes into resonance with a perturbing force arising from the motion of major bodies. Such resonances can be of two kinds: one causing stability for that particular period, the other removing material even more quickly from orbits of that period than the spiral motion would have done. In the one location in the solar system where we still see small particulate matter on long-lived orbits, we indeed see the material organized into well-defined rings, namely the rings of Saturn. It may well be that even at the present time there exists a similar banded structure for particles of a certain size range in the plane of the solar system, with some bands as a result of planetary perturbations having permanent stability, despite the Poynting-Robertson effect. The zodiacal light may be due to a set of bands that would make the solar system look like a faint version of Saturn's rings when viewed from outside the central plane. 
Such bands will have been a most important feature in the condensation processes in earlier times. It is in these circumstances, where a nonconservative force has been active, that Liouville's theorem is not satisfied, and indeed particles will be driven into much higher densities than the densities at which they were originally supplied. It is important to realize that the process will also sort particles for a certain size range, in the sense that the ones that are too small (for the strength of any particular celestial mechanics resonance) cannot be arrested against the drag force that calses spiraling, while particles that are too large may spiral so slowly as not to reach a resonant condition. A resonant band will thus first be supplied with the smallest particles that can be stable in that resonance and will later gradually acquire larger and larger objects. The relative velocities, and thus the erosion rates through mutual collisions, will become very small, and the circumstances will be favorable for snowballing, to make larger particles with the help of any surface stiction. The concentration of asteroids into the Trojan orbits cannot readily be understood without such an action. It is also important to realize that such lanes may in some cases have been supplied principally from original condensate, but in other cases from debris of collisions of earlier bodies. Different lanes may thus be chemically and mineralogically distinctive. In the course of long times, the stability of individual planetary bands may be lost as a result of various changes. Major perturbations among the planets, further accretion, or major collision, could so change the celestial mechanics situation as to destroy the resonance with a particular band, and it would then again begin to spiral. Now, however, the starting point would be an enormously more concentrated, narrow lane of highly collimated orbits, rather than a diffuse distribution in the whole disc. The densities of particulate matter in such a band may well have risen above the mean by a factor of the order of $10^{4}$. We must then visualize that there will have been many events of such highly concentrated bands becoming unlocked from their resonance and therefore spiraling in the solar system. When such material reaches the vicinity of a planet each particle may suffer one of three possible fates. Firstly it may impact the planet and thus contribute to its growth. Secondly it may, after a period of large perturbations, cross safely to the other side of the sphere of influence of that planet and then continue with its spiral. Thirdly, it may be placed on a satellitic orbit to that planet, a probability which would be greatly increased by the presence of a frictional medium such as the main solar nebula, or its temporary concentrations in the vicinity of the planets. It is extremely difficult to make any estimate of the relative probability of the three types of encounter. Even if the setting up of a satellite orbit were many times less probable than the other two possibilities, it would still suffice to lead to the eventual formation of the satellites that we know, if the process occurred early enough so that a large proportion could still be added to make the planets grow.

The step from circumplanetary rings attenuated by gas friction to the formation of satellite bodies is not a difficult one and has been discussed on many occasions. If in the first place a number of separate satellites form, as may well be the rule, it will depend on their size distribution, and the tidal friction with the planet, whether they can be maintained as separate bodies. If tidal friction is sufficient and there is a 
forward spinning planet, and if the innermost satellite formed is more massive than the others, then they will all be swept up into one body finally. This is because the most massive satellite will spiral out the fastest, and there is no possibility in this case of one body crossing the lane of the next without colliding with it. Perhaps this is the set of events that occurred to make our rather large Moon, while in the case of the major planets the evolution through tidal friction has been somewhat slower. The material that now forms the surface of the Moon is perhaps the last addition, and the soil that is found there is material acquired directly in its present form from orbit. The fact that the material has suffered chemical differentiation on a planetary body in its past does not argue against such a theory. As we have said, many of the bands will be debris from collisions, and the last material acquired by the Moon may be one of those. Whatever finely divided material fell into the Moon in the late phases would tend to make a set of layers that may be chemically distinctive. Any of the larger impacts, such as those that caused the mare basins, must then rework the layered structure into one that makes for regional differences in composition. Much detail that is now known about the lunar soil is difficult if not impossible to account for within the view that this soil resulted from the grinding up by meteorite bombardment of solid lunar rocks. In particular, the high intensity and remarkable uniformity of the cosmic ray exposure of all the soil seems to accord much better with a picture in which this soil was in diffuse form in orbit and fell in to make the last addition to the Moon. (The impact on collision of each grain with the lunar surface would not heat the particles enough to eradicate the cosmic ray tracks if infall was from other Earth satellite orbits only.)

The recent radar observations from the Jet Propulsion Laboratory at Goldstone of Saturn's rings suggest that they are made largely of metallic particles. (The alternative theory that they are made of dielectric material in accurately spherical form - cat's eyes - is considered unlikely, since we know of no mechanism that would tend to assemble meter-sized spheres as would be required.) This observation emphasizes the view that bands of particular composition can be formed and become placed on satellite orbits. The rings of Saturn are too close to the planet to form further satellites, but the greatly varying composition of the satellites suggests that similar processes had occurred there but with differently sorted out, second generation debris material.

\section{Acknowledgement}

Work on lunar studies is carried out under NASA Grant NGL-33-010-005. 